\title{
Learning to Recognize Animals by Watching Documentaries: Using Subtitles as Weak Supervision
}

\author{
Aparna Nurani Venkitasubramanian ${ }^{1}$, Tinne Tuytelaars ${ }^{2}$, and Marie-Francine Moens ${ }^{1}$ \\ ${ }^{1}$ KU Leuven, Computer Science Department, Belgium \\ ${ }^{2}$ KU Leuven, ESAT-PSI, IMEC, Belgium \\ \{firstname. lastname\} @kuleuven.be
}

\begin{abstract}
We investigate animal recognition models learned from wildlife video documentaries by using the weak supervision of the textual subtitles. This is a challenging setting, since i) the animals occur in their natural habitat and are often largely occluded and ii) subtitles are to a great degree complementary to the visual content, providing a very weak supervisory signal. This is in contrast to most work on integrated vision and language in the literature, where textual descriptions are tightly linked to the image content, and often generated in a curated fashion for the task at hand. We investigate different image representations and models, in particular a support vector machine on top of activations of a pretrained convolutional neural network, as well as a Naive Bayes framework on a 'bag-of-activations' image representation, where each element of the bag is considered separately. This representation allows key components in the image to be isolated, in spite of vastly varying backgrounds and image clutter, without an object detection or image segmentation step. The methods are evaluated based on how well they transfer to unseen camera-trap images captured across diverse topographical regions under different environmental conditions and illumination settings, involving a large domain shift.
\end{abstract}

\section{Introduction}

It is estimated ${ }^{1}$ that video traffic will be 82 percent of all global Internet traffic by 2020 . The

\footnotetext{
${ }^{1}$ http://www.cisco.com/c/en/us/solutions/collateral/serviceprovider/visual-networking-index-vni/complete-whitepaper-c11-481360.html
}

ubiquitousness of video on the web demands indexing tools that facilitate fast and easy access to relevant content. Traditionally, video search has been based on user-tags. However, in the recent past, research activities have been directed at automatic indexing of videos based on the content. Contributing to this goal of automatic video indexing, we focus on the problem of wildlife recognition in nature documentaries with subtitles.

This setup is challenging from at least two perspectives: first, from the point of view of the content, and second, due to the nature of video documentaries. As far as the content is concerned, we are dealing with animals shot in their natural habitat. The problem of identifying animals in videos, especially those shot in the natural habitat presents several challenges. Firstly, animals are among the most difficult objects to recognize in images and videos, mainly due to their deformable bodies that often self occlude and the large variation they pose in appearance and depiction (Afkham et al., 2008; Berg and Forsyth, 2006). Further, in the natural habitat, there are challenges due to camouflage and occlusion by flora. Moreover, unlike faces or cuboidal objects such as furniture, we do not have accurate detectors that can localize the animal in a frame. State-of-the-art object proposal methods such as (Girshick et al., 2014; Ren et al., 2015) yield an unacceptably low level of either recall or precision. The absence of detectors necessitates other mechanisms that allow segregation of the components of the image.

The nature of video documentaries presents yet another challenge. Typically, in video documentaries such as ours, the subtitles are not parallel, but complementary to the visuals (See Fig. 1). This is in contrast to most work on integrated vision and language in the literature, where textual descriptions are tightly linked to the image content. This means we do not have examples that 


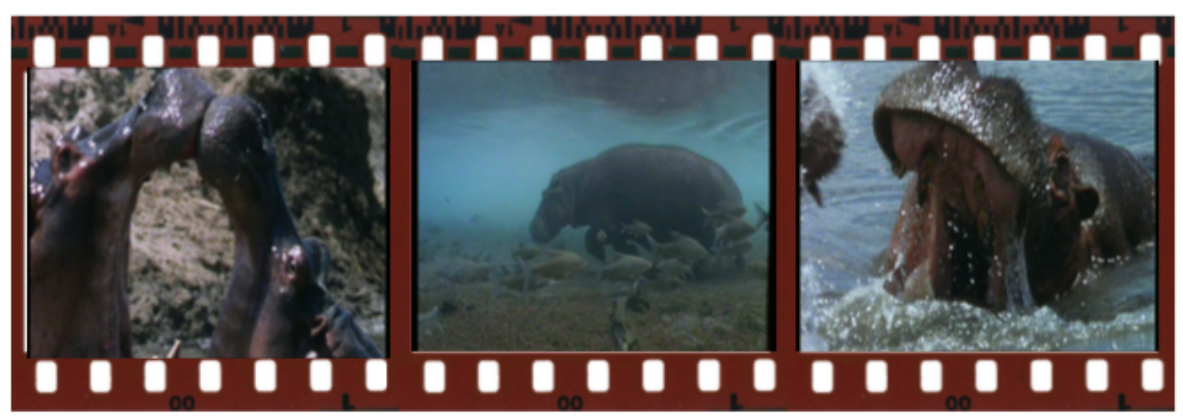

In the rivers and lakes of Africa, lives an animal which has a reputation for being the most unpredictable and dangerous of all.

Even crocodiles are wary. The hippopotamus.

Figure 1: A set of frames together with the corresponding subtitles: The frames show hippos, while the subtitles mention both hippo and crocodile.

can reliably tie together textual and visual entities.

In this work, we study image representations and models that cope with the above challenges. These include a support vector machine on top of activations of a pretrained convolutional neural network, and a Naive Bayes framework on a 'bag-of-activations' image representation, where each element of the bag is considered separately. While the former utilizes a global representation denoted by the feature vector comprising $\mathrm{CNN}$ activations, the latter works on per dimension basis, allowing key components in the image to be isolated, in spite of largely varying backgrounds and image clutter, without an object detection or image segmentation step. We experiment with both continuous and discretized variants of the 'bag-of-activations' representation. In particular, we investigate image representations and weakly supervised animal recognition models that can be learned without the need for bounding boxes, or curated data comprising manually annotated training examples.

The rest of this paper is organized as follows: Section 2 presents the background and related work. Section 3 provides the problem definition. Section 4 describes the image representations and animal recognition models based on CNN activations. Section 5 discusses the experiments and results. Finally, Section 6 provides the conclusions.

\section{Related Work}

Identifying animals is a well-studied topic (Afkham et al., 2008; Berg and Forsyth, 2006; Schmid, 2001; Ramanan et al., 2006). Recent works such as (Hariharan and Girshick, 2016) and (Gomez and Salazar, 2016) advance us further and provide better insight into the problem. However, these methods are not applicable in our setting since they require extensive training data. It is important to note that in this setup, we lack sufficient reliable training data making neural networkbased training impractical.

Apart from these works that focus specifically on animals, there is a large literature on generic object detection. These methods are often evaluated on the Pascal VOC challenge dataset (Everingham et al., 2012) which includes classes of animals such as cats, dogs, cows and horses, among other things. There are also datasets that focus on animals such as Caltech UCSD Birds (Wah et al., 2011) and Stanford Dogs (Khosla et al., 2011). Additionally, the FishClef and BirdClef challenges which are part of LifeClef (Joly et al., 2015) provide an arena for identification of species of fish and birds respectively. Most of these datasets are, however, object-centered and in that sense easier than the 'in-the-wild' setting we are dealing with.

The problem of aligning animals from videos with their mentions in subtitles has been studied in (Dusart et al., 2013) and (Venkitasubramanian et al., 2016). The former relies on hand-annotated bounding boxes to localize the animals in a frame, which are difficult to acquire. The latter relies on training animal classifiers on labeled external data such as ImageNet (Deng et al., 2009), and has the issue that not all classes of objects can be learned from an external dataset, for instance, rare species 
of animals may not be found on ImageNet.

Recently, there has been considerable interest in sentence/caption generation from images as well as natural language based object detection, e.g. (Karpathy and Fei-Fei, 2014; Fang et al., 2014; Guadarrama et al., 2013; Kazemzadeh et al., 2014). These approaches typically rely on text snippets that accurately describe the content of the images or videos. However, in our context, the subtitles and the visuals are not parallel, but complementary. For example, often a few animals are mentioned in the text, while the connected frame only shows one of them. The connection between the vision and the text is therefore much weaker. Additionally, in our setup, we have too few data to train similar models. As a result, these approaches are not directly applicable to our setting. In this paper, we explore weakly-supervised models that can deal with the complementarity or the "nonparallelism' of the visual and textual modalities.

There has also been some work on alignment across modalities for recognizing people (Pham et al., 2010, 2011; Guillaumin et al., 2008). These approaches rely on the use of a face-detector. While there are face detectors available with reasonable accuracy, there are no such detectors that allow localizing animals. The absence of the bounding boxes complicates the problem in many ways. A notable endeavor in this domain is that of (Everingham et al., 2006) where dialogue transcripts and other supervisory information (such as lip movements or clothing) are used in addition to subtitles and face detectors. In our context, since the subjects of our videos involve animals, cues such as lip movements or clothing are not relevant.

In this paper, we investigate image representations and multi-modal animal recognition models that can cope with a) complementarity of vision and language, $b$ ) lack of bounding boxes and c) lack of labeled external data, and can transfer to a different unseen domain, shot under very different conditions.

\section{Task definition}

We have a wildlife documentary with subtitles. On the visual side, we derive key frames $\mathbf{F}=\left\{f_{1}, f_{2} \ldots f_{q}\right\}$ from which we extract visual features with a suitable representation $\mathbf{A}=$ $\left\{\mathbf{a}_{1}, \mathbf{a}_{2} \ldots \mathbf{a}_{\mathbf{q}}\right\}$. Assume each feature vector has $\mathrm{D}$ dimensions. On the textual side, from the subtitles, we identify the unique animal mentions or animal names $\mathbf{N}=\left\{n_{1}, n_{2} \ldots n_{p}\right\}$, using a list of animal names derived from WordNet (Miller, 1995) as in (Dusart et al., 2013).

Using the setup of (Venkitasubramanian et al., 2016), we associate every frame $f_{i}, 1 \leq i \leq q$, with a set $\mathbb{N}_{i} \subset \mathbf{N}$ of possible animal names derived from 5 subtitles to the left and right of the frame. The set $\mathbb{N}_{i}$ refers to the set of unique animal names derived from their mentions and coreferences in the subtitles ${ }^{2}$. It is possible that the frame has some or all or none of the animals in $\mathbb{N}_{i}$. Corresponding to every name $n_{l} \in \mathbb{N}_{i}$, we have a binary label $y_{l}$ indicating the presence or absence of $n_{l}$. Our objective is to find the most likely value of $y_{l}$ corresponding to name $n_{l} \in \mathbb{N}_{i}$ for every frame $f_{i}$.

\section{Image Representations Based on CNN Activations}

A popular choice of visual features for object recognition is the activations of the penultimate layer of a pretrained Convolutional Neural Network. In this work, we use the VGG CNN-M-128 architecture $^{3}$ of (Chatfield et al., 2014), which is trained on 1,000 object categories from ImageNet (Deng et al., 2009) with roughly 1.2M training images. Within this realm, we explore two perspectives on the real-valued feature vector: (i) a global representation where each feature vector is treated as one entity, and (ii) a bag-of-activations representation, where each element of the bag is considered separately.

The global representation is by far the most commonly used (Sharif Razavian et al., 2014) and fits well with a linear Support Vector Machine (SVM) classifier. For the task of object recognition, the linear SVM is typically used with the $L_{2}$ norm, and has the following objective function

$$
\underset{\mathbf{w}_{\mathbf{l}}}{\operatorname{minimize}} \frac{1}{2}\left\|\mathbf{w}_{\mathbf{l}}\right\|^{2}+C \sum_{i} \max \left(1-y_{l} \mathbf{w}_{\mathbf{l}}^{T} \mathbf{a}_{\mathbf{i}}, 0\right)
$$

where $\mathrm{w}_{\mathbf{l}}$ denotes the set of weights to be learned for the label $y_{l}$ corresponding to name $n_{l}$, and $C$ denotes the $\operatorname{cost}^{4}$. In a weakly supervised setting, these weights are learned based on the

\footnotetext{
${ }^{2}$ There remains a small percentage $(2.35 \%)$ of animals not mentioned in the nearby subtitles. These will be left undetected.

${ }^{3}$ This model yielded 128 features.

${ }^{4}$ We used the Liblinear (Fan et al., 2008) toolkit, with the default setting of 1 for the cost $C$.
} 
weakly associated (hence noisy) frame-name pairs $<\mathbf{a}_{\mathbf{i}}, n_{l}>$ for all $n_{l} \in \mathbb{N}_{i}$.

An alternative to this global representation is a bag-of-activations representation, where each feature dimension is treated in isolation. $\mathrm{Li}$ et al. (2014) have shown that the CNN activations have two interesting properties: firstly, they can be treated independently along the dimensions and second, they preserve their essence even after binarization. We exploit the first property and use it in a naive Bayes framework. The idea of treating each element of the $\mathrm{CNN}$ representation individually rather than using the full feature vector in a high-dimensional space is crucial: It brings robustness to image clutter and changing backgrounds, and helps in learning from few examples.

$$
p\left(y_{l} \mid \mathbf{a}_{\mathbf{i}}\right)=\frac{p\left(y_{l}\right) \prod_{v=1}^{D} p\left(a_{i v} \mid y_{l}\right)}{Z_{l}}
$$

$Z_{l}$ is a normalization constant for the name $n_{l}$, given by

$$
Z_{l}=p\left(y_{l}\right) \prod_{v=1}^{D} p\left(a_{i v} \mid y_{l}\right)+p\left(\overline{y_{l}}\right) \prod_{v=1}^{D} p\left(a_{i v} \mid \overline{y_{l}}\right)
$$

where $\overline{y_{l}}=0$ if $y_{l}=1$ and vice versa. $p\left(y_{l}\right)$ is the prior which we assume to be uninformative for simplicity. So, $p\left(y_{l}=0\right)=p\left(y_{l}=1\right)$.

Then, using Eq. 2, Eq. 1 can be written as follows:

$$
p\left(y_{l} \mid \mathbf{a}_{\mathbf{i}}\right)=\frac{\prod_{v=1}^{D} p\left(a_{i v} \mid y_{l}\right)}{\prod_{v=1}^{D} p\left(a_{i v} \mid y_{l}\right)+\prod_{v=1}^{D} p\left(a_{i v} \mid \overline{y_{l}}\right)}
$$

The second interesting property of the $\mathrm{CNN}$ activations is that they preserve their essence even after binarization. We investigate this further and show that not only binarization but also discretization of the feature vector into a larger number of bins is useful. In particular, we propose to discretize the feature vector into B bins along each dimension $^{5}$. In this paper, we experiment with two approaches for binning the feature vector - (i) equal width and (ii) equal frequency. The equal width approach ensures that all the bins are of the same size. For example, if we are interested in 2 equal width bins, we could look at the feature vector along a dimension and set the threshold midway between the minimum and maximum values

\footnotetext{
${ }^{5}$ Discretization can also be applied to the global representation used by the SVM, but as shown in (Venkitasubramanian et al., 2016), it is particularly useful in conjunction with a naive Bayes classifier.
}

of that dimension. The values that are less than the threshold could be set to 0 , while the rest are set to 1 . In equal frequency binning, the threshold is set such that the number of elements in each bin is roughly the same.

This discretization is similar to the vector quantization of SIFT descriptors to obtain Bag of Visual Words (BoVW). But, while BoVW has the issue that the discretization errors can have a significant negative impact, with $\mathrm{CNN}$ features, there are no strong discretization artifacts. In fact, Li et al. (2014) have shown that retaining just the values of the largest $k$ dimensions (or even setting the values of the largest $k$ dimensions to 1 and the rest to 0 ) is sufficient to capture the essence of the image.

Discretizing the feature space allows us to replace the feature $a_{i v}$ by the corresponding bin $\beta_{v}$.

$$
p\left(a_{i v} \mid y_{l}\right)=p\left(\beta_{v} \mid y_{l}\right)
$$

where $\beta_{v} \in\{0,1 \ldots B\}$ is the bin to which $a_{i v}$ belongs.

Eq. 3 can then be rewritten as

$$
p\left(y_{l} \mid \mathbf{a}_{\mathbf{i}}\right)=\frac{\prod_{v=1}^{D} p\left(\beta_{v} \mid y_{l}\right)}{\prod_{v=1}^{D} p\left(\beta_{v} \mid y_{l}\right)+\prod_{v=1}^{D} p\left(\beta_{v} \mid \overline{y_{l}}\right)}
$$

To compute the conditional probabilities $p\left(\beta_{v} \mid y_{l}\right)$ of the bin $\beta_{v}$ given $y_{l}$, we rely on the noisy labels that can be obtained from the text. Basically we count the co-occurrence of label $y_{l}$ corresponding to name $n_{l} \in \mathbb{N}_{i}$ with bin $\beta_{v}$ relative to the total number of instances where $y_{l}$ occurs in our dataset.

$$
p\left(\beta_{v} \mid y_{l}\right)=\frac{\operatorname{freq}\left(\beta_{v}, y_{l}\right)}{\operatorname{freq}\left(y_{l}\right)}
$$

\section{Experiments and Results}

The dataset used in our experiments is that of (Dusart et al., 2013). This is a wildlife documentary named 'Great Wildlife Moments ${ }^{6}$ with subtitles from the BBC. This is an interlaced video with a duration of 108 minutes at a frame rate of 25 frames per second, and the frame resolution is $720 x 576$ pixels. The video consists of 28 chapters and all the chapters except the ones containing just one animal are evaluated. This leaves us with chapters 14 to 28 . Applying shot cut detection (Hellier et al., 2012) on these chapters, we obtained 602 key frames. Of these, 302 frames had

\footnotetext{
${ }^{6} \mathrm{https} / / /$ en.wikipedia.org/wiki/Great_Wildlife_Moments
} 


\begin{tabular}{|l|c|c|c|}
\hline Method & Precision & Recall & $F_{1}$ \\
\hline SVM & 80.43 & 12.71 & 21.96 \\
Naive Bayes & 20.23 & 71.48 & 31.54 \\
\hline
\end{tabular}

Table 1: Results of using the continuous features and applying the weak labels of our dataset

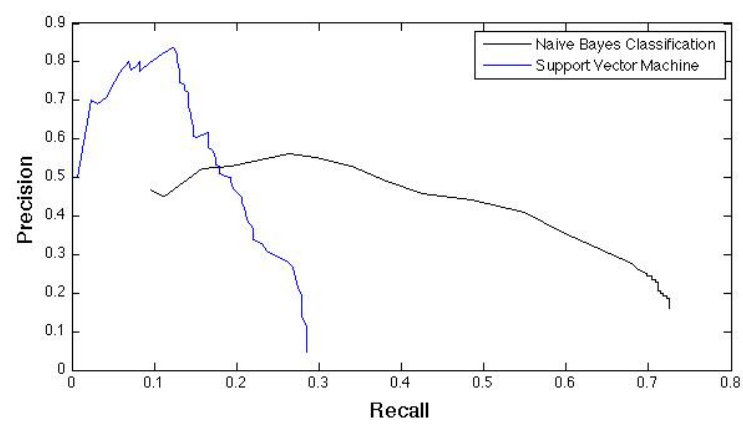

Figure 2: The precision-recall curves for the SVM and naive Bayes classifier shown in Table 1. Area under the curve is 0.1599 for the SVM and 0.3642 for naive Bayes.

no animal. The remaining 300 contained 365 animals in total. We run our algorithm on all the 602 key frames. There were 19 species of animals.

The animal labeling is evaluated in terms of precision, recall and $F_{1}$ computed over the entire dataset as follows:

$$
\begin{gathered}
\text { precision }=\frac{\text { number of labels correctly assigned }}{\text { total number of labels assigned }} \\
\text { recall }=\frac{\begin{array}{c}
\text { number of labels correctly assigned } \\
\text { actual number of animal present }
\end{array}}{}
\end{gathered}
$$

The evaluation covers two aspects:

1. How well do the representation and model learned using the weak labels of our dataset perform on the same dataset? (Section 5.1)

2. How well do the representation and model learned using the weak labels of our dataset transfer to an external dataset shot over diverse topographical regions under different environmental conditions and illumination settings? (Section 5.2)

\subsection{Animal labeling on wildlife videos}

Table 1 shows the performance of an SVM on the global representation and a naive Bayes classifier on the bag of activations using continuous features. In either case, name $n_{l}$ is assigned to frame

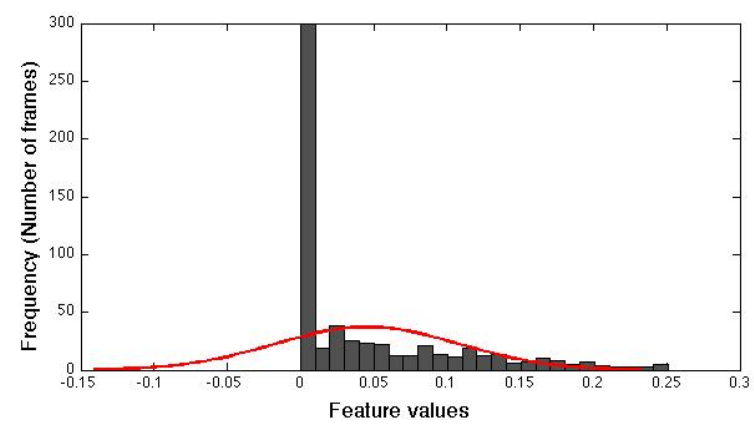

Figure 3: The distribution of the feature values along the first dimension: $\mathrm{x}$-axis shows the range of feature values, y-axis shows the number of frames. The grey histogram shows the distribution of the feature values. The red curve is the normal distribution plotted using the mean and standard deviation along the first dimension, $\mathcal{N}(0.0454,0.0622)$.

$\mathbf{a}_{\mathbf{i}}$ if $p\left(y_{l} \mid \mathbf{a}_{\mathbf{i}}\right)>p\left(\overline{y_{l}} \mid \mathbf{a}_{\mathbf{i}}\right)$, that is, the probability threshold for prediction was set at 0.5. For the naive Bayes classifier, a Gaussian distribution was used to model the continuous features along each dimension. While both models do not yield adequate performance, the naive Bayes certainly does far better compared to the SVM. In this setup involving limited reliable example pairs, it is beneficial to treat each element of the CNN representation individually rather than using the full feature vector in a high-dimensional space. Fig. 2 shows the precision-recall curves of the SVM and the naive Bayes classifier. The naive Bayes is clearly better in this setup, except in the low recall / high precision region.

Closer inspection reveals that the Gaussian distribution used in the Naive Bayes framework is not a good fit to the data (see Fig. 3 for one example feature dimension). Fig. 3 shows the normal distribution plotted using the mean and the standard deviation along the first dimension for the entire dataset (red curve: $\mathcal{N}(0.0454,0.0622)$ ). This is superimposed on the histogram of the real-valued (undiscretized) feature vector (in grey). While there are certainly other distributions (such as Poisson or Binomial) that could be used to model the data, we show that the most commonly used Gaussian clearly does not fit the data. Rather than forcing the data to fit into some distribution, we turn to a discretized setting as it allows use of a simple non-parametric model. 


\begin{tabular}{|l|c|c|c|}
\hline Method & Precision & Recall & $F_{1}$ \\
\hline$B=2$ & 46.43 & 91.55 & 61.61 \\
$B=3$ & 46.85 & 94.37 & 62.62 \\
$B=4$ & 47.03 & 92.96 & 62.46 \\
$B=5$ & 47.18 & 94.37 & 62.91 \\
$B=6$ & 47.88 & 95.31 & 63.74 \\
$B=7$ & 47.69 & 96.71 & $\mathbf{6 3 . 8 8}$ \\
$B=8$ & 47.45 & 96.24 & 63.57 \\
$B=9$ & 47.00 & 95.77 & 63.06 \\
$B=20$ & 46.47 & 95.77 & 62.58 \\
\hline $\log _{2} l$-bins & 47.47 & 96.71 & 63.68 \\
\hline
\end{tabular}

\begin{tabular}{|l|c|c|c|}
\hline Method & Precision & Recall & $F_{1}$ \\
\hline$B=2$ & 48.04 & 92.02 & 63.12 \\
$B=3$ & 47.95 & 93.43 & 63.38 \\
$B=4$ & 46.99 & 95.31 & 62.95 \\
$B=5$ & 46.24 & 95.31 & 62.27 \\
$B=6$ & 45.56 & 96.24 & 61.84 \\
$B=7$ & 45.23 & 95.77 & 61.45 \\
$B=8$ & 44.93 & 95.77 & 61.17 \\
$B=9$ & 44.81 & 97.18 & 61.33 \\
$B=20$ & 43.51 & 97.65 & 60.20 \\
\hline
\end{tabular}

Table 2: Results of using the discretized features (left: equal width discretization, right: equal frequency discretization) and applying the weak labels of our dataset

Next, we present the results of using the discretized features. Table 2 (left) shows the results of the animal labeling using equal width binning for different number of bins $B$. First, we use a fixed number of bins over every dimension. That is, along every dimension in the feature vector, the number of bins is set to a constant $B$. Note that irrespective of the number of bins, the performance has improved significantly. The precision has more than doubled, and the recall has improved by more than $20 \%$ absolute. Contrary to expectations, the discretization has actually improved the classification. These findings are consistent with those of Dougherty et al. (Dougherty et al., 1995). Overall, we see that these results are significantly better than all the baselines in Table 1. In addition to the discretization, the key aspects of this method are the use of naive Bayes classifier and the idea of treating each element of the CNN representation separately rather than using the full feature vector in a high-dimensional space. These bring robustness to image clutter and changing backgrounds, and help in learning from few examples.

Next, looking at the $F_{1}$ measures for different values of $B$, we see that the best results are obtained when $B=7$. In addition to fixing the number of bins along every dimension, we used a heuristic to set a variable number of bins for each dimension. Using the heuristic in S-Plus histogram algorithm of Spector (Spector, 1994), we set the number of bins along each dimension to $\log _{2} l$, where $l$ is the number of unique values in that dimension. Using this heuristic, different dimensions had different number of bins. We observed that of the 128 dimensions, 12 had 7 bins, while the rest had 8 bins. This explains why we have the best results in the range $B=7$ and $B=8$.

Table 2 (right) shows the results of the animal labeling using equal frequency binning for different number of bins $B$. Here, since we are dealing with sparse matrices, we have to ensure that all zero-valued entries along a dimension should belong to the same bin. The results in table 2 incorporate this correction. As with the equal-width case, we obtain significant improvements over the naive Bayes classifier with continuous features.

Fig. 4 shows some of the sample outputs of our system. Note that our method is capable of identifying multiple species in the same frame, as well as detecting frames that do not contain any animal.

\subsection{Transfer to camera-trap images}

The second aspect of the evaluation is to measure how well the representations and models transfer to external data from an entirely different setup. To evaluate this, we use the Snapshot Serengeti (Swanson et al., 2015) dataset, which consists of camera-trap (remote, automatic cameras) images covering wildlife in Savanna. We learn animal recognition models using the weak labels of our dataset and apply them to the Snapshot Serengeti (Swanson et al., 2015) dataset. It is important to note that the pictures of this Serengeti dataset are captured automatically, in very different scenes, under various illumination conditions. This causes a huge domain shift. The Serengeti dataset covers 40 mammalian species, of which three (Lion, Zebra and Hippopotamus) also appear in our dataset. We choose 500 random images ${ }^{7}$ each of Lion and

\footnotetext{
${ }^{7}$ shot between 6:00 am and 6:00 pm
} 


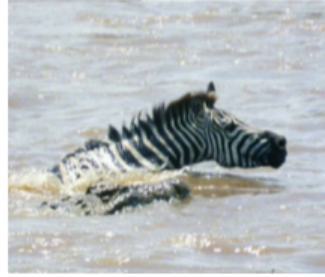

GT: Zebra, Crocodile Predicted: Zebra,

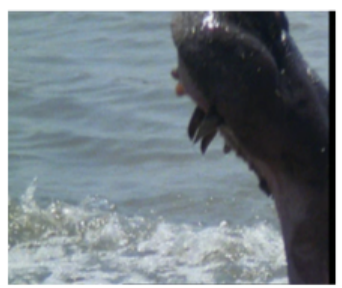

GT: Hippopotamus

Predicted: -

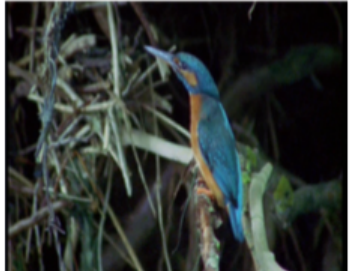

GT: Kingfisher

Predicted: Kingfisher

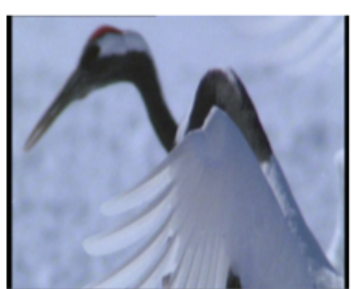

GT: Crane

Predicted: Crane

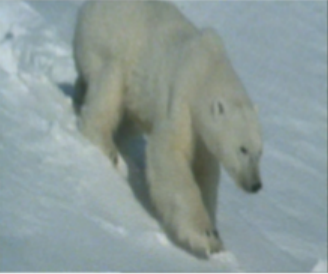

GT: Bear

Predicted: Bear, Penguin

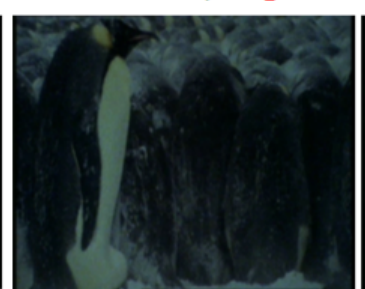

GT: Penguin

Predicted: Penguin

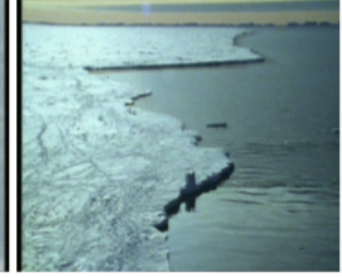

GT: -

Predicted: -

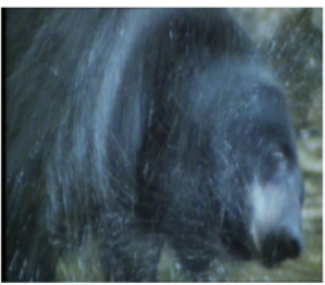

GT: Bear

Predicted: Bear

Figure 4: Some sample outputs from our system. 'GT' indicates ground truth, 'Predicted' indicates the predictions of the system.

Zebra, and all 37 images available for the Hippopotamus class. This set forms the target data on which the animal recognition models will be tested. Fig. 5 shows some of the sample images from this dataset.

Table 3 shows the performance of the animal recognition models learned using our data, applied on the target dataset. The first baseline is simply based on the probabilities output by the CNN pretrained on ImageNet. We used the same architecture (CNN-M-128) that was used for feature extraction. When the output probability for a certain class was $>0.5$, we concluded that the system predicted that class. Of course, multiple classes could be predicted for each key frame. Although some of the classes predicted covered 'lake side', 'hay' etc. which were not explicitly labeled in our setup, there were a lot of animals incorrectly predicted (which did not belong to our dataset of 19 animals). These included elephant, panther, camel, dugong. We filtered the outputs to just retain the 19 classes that were seen in our dataset. This increased the precision by a large margin (second row in the table). Next, we retained only the three classes that were common to our dataset and Serengeti dataset. While this gave a perfect precision, the recall stands low at approx. 20\% in all the three cases above.

Next, we train an SVM (on the continuous features) on all the 19 classes of our dataset, using the weak association of the subtitles and applied them to Serengeti (Swanson et al., 2015) dataset
(Second block on table 3). Note that the performance is low compared to ImageNet cases in the first block. The model learned by the SVM on our dataset does not compare well with that of ImageNet, which was trained on several thousands of zebra, hippos and lions. As with the previous block, filtering to the 3 relevant classes increases the precision by a large margin, while the recall stays the same. When we used the ground truth labels instead of the weak labels (which basically indicate if a frame could have some animal), we have a perfect precision, but the recall is even lower. By capturing elements in the background/environment which might be related to the animal, (e.g., a water body for the hippopotamus, or grasslands for the zebra), the training based on weak labels yields higher recall, albeit at the cost of precision.

The last block shows the performance using a naive Bayes, trained using both weak labels, and the ground truth. Again, we note that the precision is better with groundtruth labels, while the recall is lower. But in either case, there are remarkable improvements compared to the first and second blocks. The idea of treating each element of the CNN representation individually rather than using the full feature vector in a high-dimensional space is crucial both for isolating the object( $(s)$ of interest from the clutter, and for learning with few examples. The discretized naive Bayes does not perform better than the continuous naive Bayes in this case - the discretized features probably do not 

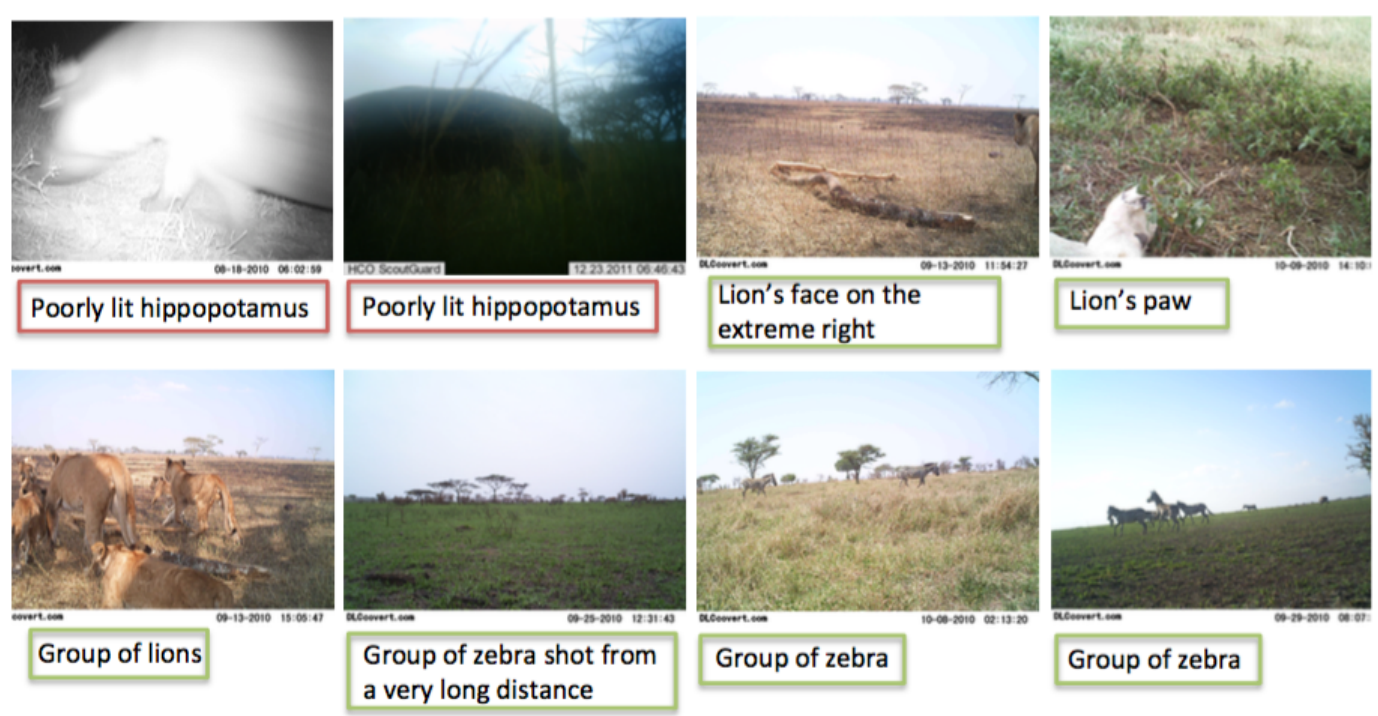

Figure 5: Some sample images from the Snapshot Serengeti (Swanson et al., 2015) dataset, together with the descriptions that show the difficulty of the task. Green box indicates the animal was recognized correctly, while red indicates the animal was missed.

\begin{tabular}{|l|c|c|c|}
\hline Method & Precision & Recall & $F_{1}$ \\
\hline CNN-M-128 (1000 classes) & 21.98 & 20.38 & 21.15 \\
CNN-M-128 (filtered to 19 classes of our dataset) & 91.75 & 20.38 & 33.35 \\
CNN-M-128 (filtered to 3 overlapping classes) & 100 & 20.38 & 33.86 \\
\hline SVM continuous (on our 19 classes) - using weak labels & 58.16 & 14.96 & 23.80 \\
SVM continuous (on 3 overlapping classes) - using weak labels & 86.34 & 14.96 & 25.50 \\
SVM continuous (on 3 overlapping classes) - using GT & 100 & 9.31 & 17.04 \\
\hline NBC continuous (on 3 overlapping classes) - using weak labels & 49.03 & 90.53 & 63.61 \\
NBC continuous (on 3 overlapping classes) - using GT & 62.07 & 67.71 & 64.77 \\
NBC discretized into $\log _{2} l$ bins (on 3 classes) - using weak labels & 53.45 & 65.73 & 58.95 \\
\hline
\end{tabular}

Table 3: Performance of the animal recognition models learned using our data, applied on images from Snapshot Serengeti (Swanson et al., 2015) dataset

transfer as well to the target domain. Nevertheless, it certainly outperforms the classifiers in the first two blocks, by a large margin.

\section{Conclusions}

In this paper, we investigate different image representations and models, including a support vector machine on top of activations of a pretrained convolutional neural network, as well as a Naive Bayes framework on a bag-of-activations image representation, where each element of the bag is considered separately. We show that the bag-ofactivations representation allows key components in the image to be isolated, in spite of largely varying backgrounds and image clutter, and eliminates the need for an object detection or image segmentation step. In contrast to most work on integrated vision and language that use curated data, the pro- posed approach deals with vision and language that are complementary.

When the source and target are of the same domain, we also found that the discretization used with a multinomial Naive Bayes classifier yields much better performance compared to continuous features with a traditional Naive Bayes classifier the precision is more than doubled and the recall is boosted by more than $20 \%$ absolute for the task of identifying animals on a challenging dataset of wildlife documentaries. Here, we have used unsupervised equal-width and equal-frequency binning of the features. In future, we wish to explore other (weakly) supervised techniques for discretization, and their transfer to other domains. The methods proposed here take us a step closer to automatic video recognition and indexing. 


\section{References}

Heydar Maboudi Afkham, Alireza Tavakoli Targhi, Jan-Olof Eklundh, and Andrzej Pronobis. 2008. Joint visual vocabulary for animal classification. In 19th International Conference on Pattern Recognition. IEEE, pages 1-4.

Tamara L. Berg and David A. Forsyth. 2006. Animals on the web. In IEEE Computer Society Conference on Computer Vision and Pattern Recognition. IEEE, volume 2, pages 1463-1470.

Ken Chatfield, Karen Simonyan, Andrea Vedaldi, and Andrew Zisserman. 2014. Return of the devil in the details: Delving deep into convolutional nets. In Proceedings of the British Machine Vision Conference.

Jia Deng, Wei Dong, Richard Socher, Li-Jia Li, Kai Li, and Li Fei-Fei. 2009. Imagenet: A large-scale hierarchical image database. In IEEE Conference on Computer Vision and Pattern Recognition. IEEE, pages 248-255.

James Dougherty, Kohavi Ron, and Sahami Mehran. 1995. Supervised and unsupervised discretization of continuous features. In Proceedings of the twelfth international conference on Machine Learning. San Francisco, CA: Morgan Kaufmann, volume 12, page 194-202.

Thibaut Dusart, Aparna Nurani Venkitasubramanian, and Marie-Francine Moens. 2013. Cross-modal alignment for wildlife recognition. In Proceedings of the 2nd ACM International Workshop on Multimedia Analysis for Ecological Data. ACM, pages 9-14.

Mark Everingham, Josef Sivic, and Andrew Zisserman. 2006. Hello! my name is... buffy"-automatic naming of characters in tv video. In Proceedings of the British Machine Vision Conference. volume 2, page 6 .

Mark Everingham, Luc Van Gool, Cristopher K. I. Williams, John Winn, and Andrew Zisserman. 2012. The PASCAL Visual Object Classes Challenge 2012 (VOC2012) Results.

Rong-En Fan, Kai-Wei Chang, Cho-Jui Hsieh, XiangRui Wang, and Chih-Jen Lin. 2008. Liblinear: A library for large linear classification. Journal of Machine Learning Research 9:1871-1874.

Hao Fang, Saurabh Gupta, Forrest Iandola, Rupesh Srivastava, Li Deng, Piotr Dollár, Jianfeng Gao, Xiaodong $\mathrm{He}$, Margaret Mitchell, John Platt, et al. 2014. From captions to visual concepts and back. arXiv preprint arXiv:1411.4952 .

Ross Girshick, Jeff Donahue, Trevor Darrell, and Jitendra Malik. 2014. Rich feature hierarchies for accurate object detection and semantic segmentation. In Proceedings of the IEEE Conference on Computer Vision and Pattern Recognition. pages 580-587.
Alexander Gomez and Augusto Salazar. 2016. Towards automatic wild animal monitoring: Identification of animal species in camera-trap images using very deep convolutional neural networks. arXiv preprint arXiv:1603.06169.

Sergio Guadarrama, Niveda Krishnamoorthy, Girish Malkarnenkar, Subhashini Venugopalan, Raymond Mooney, Trevor Darrell, and Kate Saenko. 2013. Youtube2text: Recognizing and describing arbitrary activities using semantic hierarchies and zero-shot recognition. In IEEE International Conference on Computer Vision. IEEE, pages 2712-2719.

Matthieu Guillaumin, Thomas Mensink, Jakob Verbeek, and Cordelia Schmid. 2008. Automatic face naming with caption-based supervision. In IEEE Conference on Computer Vision and Pattern Recognition. IEEE, pages 1-8.

Bharath Hariharan and Ross Girshick. 2016. Lowshot visual object recognition. arXiv preprint arXiv:1606.02819.

Pierre Hellier, Vincent Demoulin, Lionel Oisel, and Patrick Pérez. 2012. A contrario shot detection. In 19th IEEE International Conference on Image Processing. IEEE, pages 3085-3088.

Alexis Joly, Hervé Goëau, Hervé Glotin, Concetto Spampinato, Pierre Bonnet, Willem-Pier Vellinga, Robert Planqué, Andreas Rauber, Simone Palazzo, Bob Fisher, et al. 2015. Lifeclef 2015: multimedia life species identification challenges. In International Conference of the Cross-Language Evaluation Forum for European Languages. Springer, pages 462-483.

Andrej Karpathy and Li Fei-Fei. 2014. Deep visualsemantic alignments for generating image descriptions. arXiv preprint arXiv:1412.2306.

Sahar Kazemzadeh, Vicente Ordonez, Mark Matten, and Tamara L. Berg. 2014. Referit game: Referring to objects in photographs of natural scenes. In Proceedings of the Conference on Empirical Methods in Natural Language Processing.

Aditya Khosla, Nityananda Jayadevaprakash, Bangpeng Yao, and Fei-fei Li. 2011. Novel dataset for fine-grained image categorization. In First Workshop on Fine-Grained Visual Categorization, IEEE Computer Society Conference on Computer Vision and Pattern Recognition. Citeseer.

Yao Li, Lingqiao Liu, Chunhua Shen, and Anton van den Hengel. 2014. Mid-level deep pattern mining. arXiv preprint arXiv:1411.6382.

George A. Miller. 1995. WordNet: A lexical database for English. Communications of the ACM 38:39-41.

Phi The Pham, Marie-Francine Moens, and Tinne Tuytelaars. 2010. Cross-media alignment of names and faces. IEEE Transactions on Multimedia 12(1):13-27. 
Phi The Pham, Tinne Tuytelaars, and Marie-Francine Moens. 2011. Naming people in news videos with label propagation. IEEE Multimedia 18(3):44-55.

Deva Ramanan, David A Forsyth, and Kobus Barnard. 2006. Building models of animals from video. IEEE Transactions on Pattern Analysis and Machine Intelligence, 28(8):1319-1334.

Shaoqing Ren, Kaiming He, Ross Girshick, and Jian Sun. 2015. Faster R-CNN: Towards real-time object detection with region proposal networks. In $A d$ vances in Neural Information Processing systems. pages 91-99.

Cordelia Schmid. 2001. Constructing models for content-based image retrieval. In Proceedings of the IEEE Computer Society Conference on Computer Vision and Pattern Recognition,. IEEE, volume 2, pages II-39.

Ali Sharif Razavian, Hossein Azizpour, Josephine Sullivan, and Stefan Carlsson. 2014. CNN features offthe-shelf: an astounding baseline for recognition. In Proceedings of the IEEE Conference on Computer Vision and Pattern Recognition Workshops. pages 806-813.

Phil Spector. 1994. An introduction to S and S-PLUS. Duxbury press: Wadsworth, Inc .

Alexandra Swanson, Margaret Kosmala, Chris Lintott, Robert Simpson, Arfon Smith, and Craig Packer. 2015. Snapshot Serengeti, high-frequency annotated camera trap images of 40 mammalian species in an African savanna. Scientific Data 2:150026.

Aparna Nurani Venkitasubramanian, Tinne Tuytelaars, and Marie-Francine Moens. 2016. Wildlife recognition in nature documentaries with weak supervision from subtitles and external data. Pattern Recognition Letters, Elsevier 81:63-70.

Catherine Wah, Steve Branson, Peter Welinder, Pietro Perona, and Serge Belongie. 2011. The CaltechUCSD birds-200-2011 dataset, Technical Report CNS-TR-2011-001, California Institute of Technology . 